\title{
Cognitive Problems Subscale
}

National Cancer Institute

\section{Source}

National Cancer Institute. Cognitive Problems Subscale. NCI Thesaurus. Code C157699.

A subscale of the Pediatric Quality of Life Brain Tumor Module designed to assess

cognitive problems. 